\title{
Ameliorating Effect of Terminalia arjuna Against 5-Fluorouacil Induced Cardiotoxicity in Wistar Rats
}

\section{Sushma Ghadigaonkar ${ }^{*}$, A Gopala Reddy², BDP Kala Kumar ${ }^{3}$ and M Lakshman $^{4}$}

${ }^{1}$ Assistant Professor, Department of Veterinary Pharmacology and Toxicology,

Mumbai Veterinary College, Mumbai, MAFSU, Nagpur, India

${ }^{2}$ Professor and University Head and Registrar of PV Narsimha Rao Telangana

Veterinary University, Rajendranagar, Hyderabad, India

${ }^{3}$ Professor, Department of Veterinary Pharmacology, C.V. Sc., Rajendranagar, India

${ }^{4}$ Professor and University Head, Department of Veterinary Pathology, C. V. Sc,

Rajendranagar, Hyderabad, India

*Corresponding Author: Sushma Ghadigaonkar, Assistant Professor, Department of Veterinary Pharmacology and Toxicology, Mumbai Veterinary College, Mumbai, MAFSU, Nagpur, India.

\author{
Received: July 15, 2021
}

Published: August 26, 2021

(C) All rights are reserved by Sushma

Ghadigaonkar., et al.

\section{Abstract}

The Terminalia arjuna has traditionally been used as cardiotonic. The aim of the study was to evaluate the protective effect of aqueous extract of Terminalia arjuna on pathological changes after 5-FU administration. Wistar rats where randomly divided into 6 groups: normal control, TRLE alone, Captopril alone, 5-FU alone, 5-FU + TALE and 5-FU + Captopril. Animals were sacrificed and tissue samples from the cardiac muscle were taken and processed for thickness of left ventricle wall measurement, myocardial antioxidant parameters and also for both Light and Electron Microscopical examination. 5-FU significantly altered all the parameters in the study while animals received TALE and Captopril along with 5-FU revealed significant improvement in all the parameters and the values were comparable to the control group. Light microscopic examination revealed that administration of 5-FU caused variable signs of cardiotoxicity which are represented by dilatation of cardiac muscle fiber, focal atrophy of cardiomyocyte with severe interstitial edema, and dilated blood vessels. Ultrastructural examination of these specimens confirmed the light microscopic findings and demonstrated irregular nuclear envelope, dilated myofibrils with degenerating nucleus, dilatation of nucleus, dilatation of sarcoplasmic reticulum, disorganized, electron-dense and bizarre shaped mitochondria and congestion of blood capillaries. Pre-treatment with TALE and its concomitant administration with 5-FUfor 14 days attenuated 5-FU induced myocardial damage and effectively reverted the abnormal structural changes near to normalcy. In conclusion, these results suggest that Terminalia arjuna has a protective potential in ameliorating 5-FU induced cardiotoxicity.

Keywords: Terminalia arjuna; WHO; Captopril; Wistar Rats

\section{Background}

According to the WHO (World Health Organization), Cardiovascular diseases are the leading causes of death worldwide, account- ing for an estimated 17.8 million death per year and will become even more prevalent with the recent changes in lifestyle, food habits, environmental pollution and use of the chemotherapeutic agent 
for cancer treatment [1]. Cardiac toxicity is one of the most dreaded side-effects of chemotherapeutic agents so that the gain in life expectancy due to chemotherapy might be countered by increased mortality due to cardiac problems viz., heart failure, myocardial ischemia, arrhythmias, hypertension, and thromboembolism [2,3]. There are various chemotherapeutic agents like anthracyclines [4], 5-Fluorouracil and its prodrug [5], capecitabine, cisplatin, paclitaxel and docetaxel $[6,7]$, which cause cardiotoxicity. Cardiotoxicity can appear soon after or long after chemotherapy and may vary from subclinical heart muscle dysfunction to heart failure $[6,8,9]$. Oxidative stress is a central mechanism underlying the cardiotoxicity of many chemotherapy agents [10].

\section{Introduction}

5-Fluorouracil (5-FU) is an antimetabolite Fluoropyrimidines analog of the nucleoside pyrimidine with antitumor activity. The 5-Fluorouracil forms the foundation of a variety of chemotherapy regimens and is the third most commonly used chemotherapeutic agent in the treatment of solid malignancies across the world. 5-FU is widely used systemically for breast, gastrointestinal, pancreatic and skin cancers [5]. 5-FU is the second most common therapeutic drug associated with cardiotoxicity after anthracyclines, which can manifest as chest pain, myocardial infarction or death. 5-Fluorouracil, a widely used chemotherapeutic agent, has direct toxic effects on vascular endothelium that involves endothelial nitric oxide (NO) synthase and leads to coronary spasms and endothelium-independent vasoconstriction via protein kinase $C$ [11]. The main mechanism of action is interfering with DNA synthesis and mRNA transcription. However, cardiotoxicity is one of the most important associated side effects resulting from its nonspecific Cytotoxicity in cancer cells [12]. Up to $70 \%$ of the side effects have been noted to occur within the first three days of the first cycle of 5-FU. Left ventricular dysfunction (LVD) and heart failure (HF) are two of the most serious complications of cancer treatment. Cardiotoxicity is becoming one of the most important complications of cancer chemotherapy and, sometimes, of cancer chemoprevention.

The hypothesis which was proposed that 5-FU induced cardiotoxicity could be related to oxidative stress leading to lipid peroxidation raised the possibility of using antioxidants for protectionagainst5-FU induced cardiotoxicity. The plant, Terminalia arjuna Roxb is commonly known as arjuna belongs to the family of Combretaceae. It is found in the Indo-sub Himalayan tracts of Uttar Pradesh, Southern Bihar, Madhya Pradesh, Delhi and the Deccan region. It was Vagabhatta who, for the first time, advocated the use of stem bark powder in heart ailments. The stem bark of the plant is generally used for medicinal purposes, is described in Ayurveda terms as being acrid, sweet, cooling, styptic, tonic and febrifuge in nature [13]. Fresh leaf juice is used for the treatment of earache and bark powder for treating heart ailments by the Malabar tribe, Kerala [14]. Terminalia arjuna helps in maintaining the cholesterol level at the normal rate, as it contains the antioxidant properties similar to that of vitamin E. It improves the functioning of cardiac muscle and is used in the treatment of coronary artery disease, heart failure, angina and hypercholesterolemia. Terminalia arjuna Roxb contains high levels of the antioxidant compounds including glycosides, flavonoids, tannins and inorganic minerals. Its bark powder possesses asthma relieving, diuretic, prostaglandin enhancing and coronary risk factor modulating properties [15]. It is used in the treatment of fractures, ulcers, hepatic dysfunction and showed hypercholesterolemic, antibacterial, antioxidant, antiallergic and anti-fertility activities. It is reported to possess strong hydrolipidemic properties.

This study was designed to investigate the possible ameliorative effect of Terminalia arjuna on 5-FU induced cardiac injury.

\section{Material and Methods}

Chemicals

5-FU was purchased from Neon Laboratories Limited, Andheri (E), Mumbai and was given intraperitoneally at a dose of $20 \mathrm{mg} / \mathrm{kg}$. This dose was guided by previous studies [16,17]. Captopril was purchased from Sigma-Aldrich Chemical Co., St. Louis, MO, USA and was administered by oral gavage at a dose of $20 \mathrm{mg} / \mathrm{kg}$ according to Shin., et al. [18] and Hala [16].

\section{Experimental animals}

The animals were acclimatized to experimental conditions for seven days before the start of the experiment. Rats were selected based on the body weight and randomly distributed to different groups such that the mean body weight variations were not $>20 \%$. A total of 48 animals were randomized into experimental and control groups. They were divided into 8 groups with 6 animals in each group.

\section{Experimental protocols}

- Group 1 (Control): The animals were given normal saline (2 $\mathrm{ml} / \mathrm{kg}$ b.wt./day orally), parallel to the drug-treated groups, throughout the study of 14 days. 
- $\quad$ Group 2 (TALE alone): The animals received leaves extract of TALE alone (250 $\mathrm{mg}$ in $2 \mathrm{ml}$ normal saline per $\mathrm{kg}$ b.wt.) once daily by oral intubation for 14 days.

- Group 3 (Captopril alone): The animals received captopril alone (20 mg in $2 \mathrm{ml}$ normal saline per kg b.wt.) once daily by oral intubation for 14 days.

- Group 4 (Toxic control): The animals first receive normal saline ( $2 \mathrm{ml} / \mathrm{kg}$ b.wt./day) by oral intubation for 9 days, and subsequently received 5-FU $20 \mathrm{mg}$ in $2 \mathrm{ml}$ normal saline per kg b.wt.) once daily by intraperitoneal injection for additional 5 days.

- Group 5 (5FU+ TALE): The animals first received leaves extract of Terminalia arjuna alone $(250 \mathrm{mg}$ in $2 \mathrm{ml}$ normal saline per kg b.wt.) once daily by oral intubation for 9 days and subsequently received 5-Fluorouracil once daily by intraperitoneal injection in association with TALE for additional 5 days.

- Group 6 (5-FU + Captopril): The animals first received Captopril alone at a dose of (20 mg/kg b.wt./day) by oral intubation for 9 days, and subsequently received 5-FU $(20 \mathrm{mg} / \mathrm{kg}$ b.wt./day) by intraperitoneal injection along with Captopril for additional 5 days.

At the end of the experimental period, the animals were sacrificed 24 hours after the last dose of different administrations, the chest of rats was opened and the heart specimens were rapidly removed ad immediately processed for histopathological and Ultrastructural studies.

\section{Light microscopic (LM) study}

The heart tissues were collected from the rats that were sacrificed at the end and fixed in 10\% neutral buffered formalin (NBF) for histopathological studies. The fixed tissues were processed and stained with Haematoxylin and Eosin (H\&E) stain as described by Singh and Sulochana [19].

\section{Transmission electron microscopy (TEM) of heart}

For electron microscopic studies, small heart samples were transferred to vials and fixed in $3 \%$ glutaraldehyde in $0.05 \mathrm{M}$ phosphate buffer ( $\mathrm{pH} 7.2$ ) for $24 \mathrm{~h}$ at $4^{\circ} \mathrm{C}$ and post fixed in $2 \%$ aqueous osmium tetroxide in the same buffer for $1 \mathrm{~h}$. After the post-fixation, samples were dehydrated in a series of graded alcohol and infiltrated and embedded in Araldite 6005 resin. Ultra-thin sections (50-70nm thickness) were cut with a glass knife on a Leica Ultra cut UCT-GA-D/E-1/00 ultramicrotome and mounted on grids. Then the sections were stained with saturated aqueous uranyl acetate and counterstained with $4 \%$ lead citrate [20] and were observed at various magnifications under a transmission electron microscope (Model Hitachi, H-7500) at RUSKA Lab, College of Veterinary Science, Rajendranagar, Hyderabad, India.

\section{Results and Discussions}

Light microscopic examination

The histopathology of left ventricle wall after the experimental period of 14 days is illustrated in figure (A to F). Cardiac muscle fibers of the left ventricle sections showed normal architecture in the normal control group 1 . Sections of cardiac muscle fiber of left ventricle from 5 -FU control group 5 showed dilatation of cardiac muscle fiber, focal atrophy of cardiomyocyte with severe interstitial edema, and dilated blood vessels. The focal inflammatory, cell infiltration in between damaged myocardiocytes, atrophy, splitting disruption and hyalinised of cardiomyocyte in a focal manner was also observed. Sections of cardiac muscle fiber of the left ventricle of all the treatment control groups showed no notable abnormal histological changes compared to 5-FU toxic control group. Heart sections of rats treated with 5-FU+ TALE and 5-FU + Captopril showed morphology similar to that of control 5-FU + TALE exhibited reduced damage to cardiac architecture with fewer areas of congestion along with vacuolization. However, there was congestion of blood capillaries, swelling around the nuclei and focal haemorrhage in between the myocardial bundle, partial preservation of myofibrils and decreased breaks in muscle fibers, myofibrillar vacuolization and marked cellular infiltration.

\section{Transmission electron microscopy (TEM)}

The Ultrastructural findings on left ventricle wall after the experimental period of 14 days are illustrated in figure (A to F). Ultrastructural examination of the heart tissue of the control group revealed the normal structure of cardiac muscle fibers, which are connected end to end and side to side to one another by the intercalated discs, dilated myofibrils with normal nucleus and fibrin. Mitochondria are arranged parallel to and in between the cardiac myofibrils and are found around the euchromatic nucleus. Ultrastructural examination of the left ventricular myocardium of TALE and Captopril treated groups confirmed the light microscopic findings and demonstrated dilated myofibrils with normal nucleus mild sarcoplasmic vacuolation and little myofibrillar damage. 
Ultrastructural examination of left ventricular myocardium of 5-FU toxic control group confirmed the light microscopic findings and demonstrated irregular nuclear envelope, dilated myofibrils with degenerating nucleus, dilatation of nucleus, dilatation of sarcoplasmic reticulum, disorganized, electron-dense and bizarre shaped mitochondria and congestion of blood capillaries. Ultrastructural examination of the left ventricle of groups treated with 5-FU + TALE and 5-FU + Captopril revealed myofilaments and nuclei appear somewhat normal. Degeneration of endothelial cells and undifferentiated myofibril looks like degenerated mass. The normal orientation of myofibril and architecture was found.
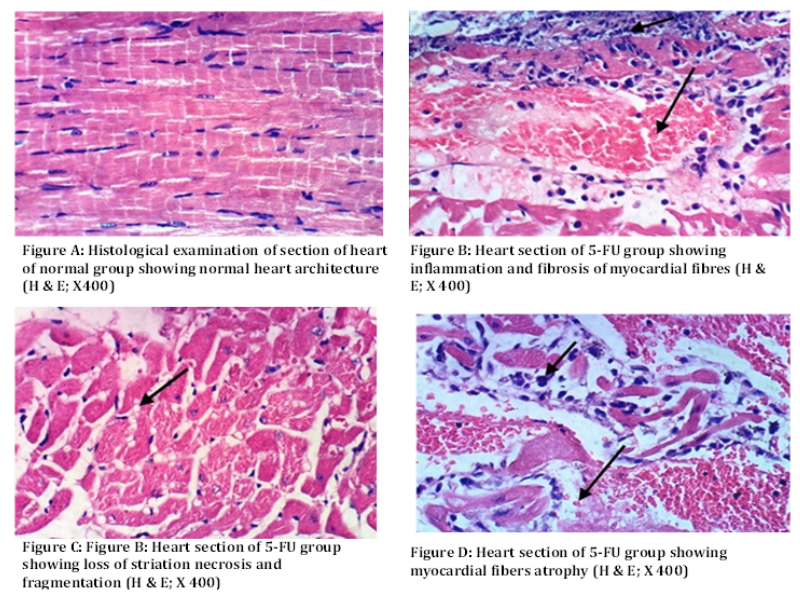

Figure 1: Pyrolysis products from microwave pyrolysis of agro-residue.

The histopathological changes of heart brought about by 5-FU in the rats revealed deviations from the normal cardiac histo-morphological features. 5-FU caused extensive damage to the cardiac musculature defined by the separation of myofibrils and extensive vacuolization with extensive muscle damage. The extent of damage with 5-FU administration was countered effectively by prior treatments with FRLE, TALE and Captopril, suggesting cardioprotective effect against 5-FU. The amelioration of biochemical changes is also supported by histopathological and electron microscopy studies in the present experiment. These results are consistent with previous studies reported by Eman and Ghada [21] confirmed that myofibrillar breaks and detachment of muscle strands accompanied by degeneration of muscle fibers due to 5 -FU treatment. Captopril is considered a standard drug for chemotherapy-induced cardiotoxicity. Hala [16] reported that pre-treatment with Captopril and its concomitant administration with 5-FU for 14days attenuated 5-FU induced myocardial damage and effectively reverted the abnormal structural changes near to normal.

\section{Conclusion}

It may be concluded from the present study, Terminalia arjuna leaf extract has a protective potential in ameliorating 5-FU induced cardiotoxicity. 5-FU exposure results in pronounced oxidative stress and administration of TALE protects the heart by scavenging free radicals.

\section{Acknowledgement}

The authors gratefully acknowledge Associate Dean, C.V.Sc., Rajendranagar and Dr. Gulam Mohammad Hussain, Research Officer (Pharmacology) Dept of Pharmacology, National Research Institute of Unani Medicine for skin Disorders, Under CCRUM, Ministry of AYUSH, Hyderabad.

\section{Declaration of Interest}

The authors report no declarations of interest.

\section{Bibliography}

1. World Health Organization (WHO): Who reveals leading cause of death and disability worldwide 2000-2019, News release, Geneva (2020).

2. Gharib I and Burnett A K. "Chemotherapy induced cardiotoxicity: current practices and prophylaxis". European Journal of Heart Failure 4 (2002): 235-242.

3. Boveli D., et al. "Cardiotoxicity of chemotherapeutic agent and radiotherapy related heart disease". Annals of Oncology 21 (2010): 277-282.

4. Van Dalen EC., et al. "Cardioprotective intervention for cancer patients receiving anthracyclines". Cochrane Library: Cochrane Reviews 2 (2011): cd003917.

5. Sorrentino M F., et al. "5-Fluorouracil induced cardiotoxicity: review of the literature". Cardiology Journal 19 (2012): 453458.

6. Stewart T., et al. "Cardiotoxicity with 5-Fluorouracil and capecitabine: more than just vasospastic angina". Internal Medicine Journal 40 (2010): 3030-307. 
7. Molinaro M., et al. "Recent advances on pathophysiology, diagnostic and therapeutic insight in cardiac dysfunction induced by antineoplastic drugs". Biomedical Research International (2015): 138-148.

8. Lenihan D J and Cardinale D M. "Late cardiac effects of cancer treatment”. Journal of Clinical Oncology 30 (2012): 3657-3666.

9. Kalam K and Marwick T H. "Role of cardioprotective therapy for prevention of cardiotoxicity with chemotherapy". European Journal of Cancer 49 (2013): 2900-2909.

10. Sterba M., et al. "Oxidative stress, redox signaling and metal chelation in anthracyclines cardiotoxicity and pharmacological cardioprotection". Antioxidant and Redox Signaling 18 (2013): 899-929.

11. Alter P., et al. "Cardiotoxicity 5-Fluorouracil". Cardiovascular and Hematological Agents Medicinal Chemistry 4 (2006): 1-5.

12. Alvarez P., et al. "5-Fluororuracil derivative: a patent review". Expert Opinion on Therapeutic Patents 22 (2012): 107-123.

13. Tyler V and Premila M S. "Ayurvedic Herbs: A clinical guide to the healing plants of traditional Indian Medicine" (2006).

14. Yesodharan $\mathrm{K}$ and Sujana K A. "Ethnomedical knowledge among Malamalasar tribe of Parambikulam wildlife sanctuary Kerala". Indian Journal of Traditional Knowledge 6.3 (2006): 481-485.

15. Chandan K., et al. "Phytochemical properties of total antioxidant status of acetone and methanol extract of Terminalia arjuna Roxb bark and its hypoglycemic effect on Type-II diabetic albino rats". Indian Journal of Pharmaceutical Science 2 (2013): 199-201.

16. Hala F Abd-Ellah. "Ameliorative effect of Captopril against 5-Fluorouracil induced cardiotoxicity in rats: A study with light and electron microscopes". Journal of Applied Sciences Research 8 (2012): 863-872.

17. Batta H and Abd E L Azim. "Biochemical studies of Captopril against 5-Fluorouracil induced heart toxicity in rats". International Journal of Advanced Research 3 (2015): 247-321.

18. Shin Chang Yong., et al. "Synergistic decrease in blood pressure by Captopril combined with Losartan in spontaneous hyper- tensive rats". Archives of Pharmaceutical Research 32.6 (2009): 955-962.

19. Singh C., et al. "Evaluation of antioxidant activity of Terminalia arjuna leave extract". Pharmacologyonline 1 (2011): 9981006.

20. Bozzollo J J and Russel L D. "Electron Microscopy: Principle and technique for biologists". Jones and Bartlett, Boston (1999): 670 .

21. Eman T M and Ghada M S. "Evaluation of cardioprotective activity of Leptidium sativum seed powder in albino rats treated with 5-Fluorouracil". Beni-Suef University of Basic and Applied Science 5 (2012): 208-215.

Volume 3 Issue 10 October 2021 (C) All rights are reserved by Sushma Ghadigaonkar., et al. 